\title{
Efficacy and Tolerability of Aripiprazole: A 26-Week Switching Study from Oral Antipsychotics
}

\author{
Jung-Sun Lee ${ }^{1}$, Seockhoon Chung ${ }^{1}$, Joon-Noh Lee ${ }^{2}$, Jun Soo Kwon ${ }^{3}$, Do Hoon Kim4, Chul Eung Kim \\ Kang Seob Oh ${ }^{6}$, Yang-Whan Jeon ${ }^{7}$, Min-Soo Lee ${ }^{8}$, Myung Ho Lim ${ }^{9}$, Hye-Ryein Chang ${ }^{10}$ and Chang Yoon Kim ${ }^{1}$ \\ ${ }^{1}$ Department of Psychiatry, University of Ulsan College of Medicine, Asan Medical Center, Seoul, Korea \\ ${ }^{2}$ Department of Psychiatry, Seoul National Hospital, Seoul, Korea \\ ${ }^{3}$ Department of Psychiatry, Seoul National University College of Medicine, Seoul, Korea \\ ${ }^{4}$ Department of Psychiatry, Hallym University College of Medicine, Chuncheon, Korea \\ ${ }^{5}$ Department of Psychiatry, College of Medicine, Inha University, Incheon, Korea \\ ${ }^{6}$ Department of Psychiatry, Kangbuk Samsung Hospital, Sungkyunkwan University School of Medicine, Seoul, Korea \\ ${ }^{7}$ Department of Psychiatry, Our Lady of Mercy Hospital, The Catholic University of Korea College of Medicine, Seoul, Korea \\ ${ }^{8}$ Department of Psychiatry, Korea University College of Medicine, Seoul, Korea \\ ${ }^{9}$ Department of Psychiatry, College of Medicine, Dankook University, Cheonan, Korea \\ ${ }^{10}$ Yong-In Mental Hospital, Yongin, Korea
}

Objective To determine if the maintenance effectiveness and tolerability of aripiprazole demonstrated in a 12-week study were maintained in an extension phase (up to 26 weeks).

Methods This study was the extension of our switching study from other antipsychotics to aripiprazole in symptomatically stable patients with schizophrenia or schizoaffective disorder. All the patients were randomly assigned to the aripiprazole group or the non-aripiprazole group. The effectiveness analysis consisted of the comparison of the upper bound of the $95 \%$ confidence interval (CI) of the mean Clinical Global Impression-Improvement (CGI-I) score to 4 (no change) at the end of the study.

Results At the baseline, the aripiprazole group $(n=135)$ and the non-aripiprazole group $(n=31)$ were comparable with respect to their mean ages, gender distribution, baseline Positive and Negative Syndrome Scale scores, and Clinical Global Impression-Severity (CGI-S) scores. The study showed that the mean CGI-I score was 2.92 (95\% CI: 2.72-3.12) in the aripiprazole group and 2.81 (95\% CI: 2.35-3.26) in the non-aripiprazole group at 26 weeks. In the aripiprazole group, the remission rates at 12 and 26 weeks were $74.8 \%$ and $72.6 \%$, respectively, and $80.2 \%$ of the patients with remission at 12 weeks maintained their remission state until the end of the study. About one-fourth of the patients in the aripiprazole group reported one or more spontaneous treatment-emergent adverse events, such as insomnia, headache, and nausea.

Conclusion This study suggested that most clinically stable outpatients with schizophrenia maintain their remission states after being switched to aripiprazole, without serious symptom aggravation and adverse events over a course of 26 weeks.

Psychiatry Investig 2010;7:189-195

Key Words Aripiprazole, Maintenance, Switch, Schizophrenia, Schizoaffective disorder.

\section{INTRODUCTION}

Aripiprazole, an atypical antipsychotic, is known to be effective for the maintenance treatment as well as acute treatments

Received: November 17, 2009 Revised: February 22, 2010

Accepted: March 17, 2010 Available online: July 9, 2010

$\triangle$ Correspondence: Chang Yoon Kim, MD

Department of Psychiatry, University of Ulsan College of Medicine, Asan Medical Center, 388-1 Pungnap-dong, Songpa-gu, Seoul 138-736, Korea

Tel: +82-2-3010-3410, Fax: +82-2-485-8381, E-mail: cykim@amc.seoul.kr

(a) This is an Open Access article distributed under the terms of the Creative Commons Attribution Non-Commercial License (http://creativecommons.org/licenses/by$\mathrm{nc} / 3.0$ ) which permits unrestricted non-commercial use, distribution, and reproduction in any medium, provided the original work is properly cited. of schizophrenia. Aripiprazole has been demonstrated to be safe and efficacious in previous studies, including short-term studies, ${ }^{1,2}$ a 26 -week placebo-controlled clinical trial, ${ }^{3}$ and a longterm, 52-week, haloperidol-controlled study. ${ }^{4}$ According to the results of a 52-week, open-label extension to a 26-week, double-blind, randomized, placebo-controlled study in patients with schizophrenia, aripiprazole showed a similar efficacy to olanzapine for the long-term treatment of acutely psychotic and chronic, stable schizophrenia patients. ${ }^{5}$

Aripiprazole has also been demonstrated to have a relatively low rate of extrapyramidal symptoms (EPSs) and a low propensity for weight gain and prolactin-related adverse events. ${ }^{1,4-8}$ 
These study findings suggest that aripiprazole may be an appropriate drug for the maintenance treatment of schizophrenia.

One of methods to investigate the maintenance efficacy of antipsychotics is to examine the changes in the symptoms or adverse events after switching from one antipsychotic to another in patients with stable symptoms. To investigate the maintenance effectiveness of aripiprazole, we conducted a prospective, non-comparative, open-label study switching from a previous antipsychotic agent to aripiprazole in schizophrenic Korean patients who were symptomatically stable. The early $12-$ week results of the study demonstrated that majority of the patients were successfully switched from their previous antipsychotics to aripiprazole without serious symptom exacerbation or adverse events over a course of 12 weeks. ${ }^{9}$

The aims of this paper were to evaluate whether the previously published results of a 12-week study on the maintenance effectiveness and tolerability of aripiprazole would be maintained up to 26 weeks in the patients who consented to participate in a 12 -week extension study.

\section{METHODS}

\section{Study design}

The design of this study was similar to those of the Broad Effectiveness Trial with Aripiprazole (BETA) and EU-BETA studies, ${ }^{10,11}$ except that the study duration was 26 weeks rather than 8 weeks.

This randomized, open-label, multicenter, 26-week study was designed to evaluate the maintenance efficacy and tolerability of aripiprazole when symptomatically stable patients with schizophrenia or schizoaffective disorder were switched from their previous prescribed oral antipsychotics to aripiprazole. The trial was conducted at 26 centers throughout Korea.

\section{Subjects}

The subjects who met all the following criteria were eligible for this study: 1 ) age: $\geq 18$ years and $\leq 65$ years; 2 ) diagnosis of schizophrenia or schizoaffective disorder (Diagnostic and Statistical Manual of Mental Disorders, Fourth Edition, DSMIV criteria) requiring long-term antipsychotic treatment; 3) symptomatically stable on a constant dose of antipsychotics for at least one month; and 4) non-admission to a hospital for at least three months prior to the study. The subjects were excluded from this study if they: 1) were undergoing their first treatment with an antipsychotic agent; 2) had taken clozapine during the last three months; 3 ) had unstable medical conditions; 4) had a history of tardive dyskinesia or neuroleptic malignant syndrome; or 5) were pregnant or breastfeeding.

All the patients who were assigned to the aripiprazole group were prescribed a starting dose of $10 \mathrm{mg}$, with gradual dose adjustment allowed within a range of 10-30 mg/day at the discretion of the investigator's judgment during the 26-week trial. The patients who were randomized to the non-aripiprazole group were treated with medications that they had not received in their prior treatment, in accordance with the generally accepted clinical practice. The patients were gradually discontinued from their previous antipsychotic medication during the first two weeks after randomization.

Of the 292 randomized patients, 186 (63.7\%) completed the previous 12-week trial (Fig. 1). At 12 weeks (baseline of the ex-

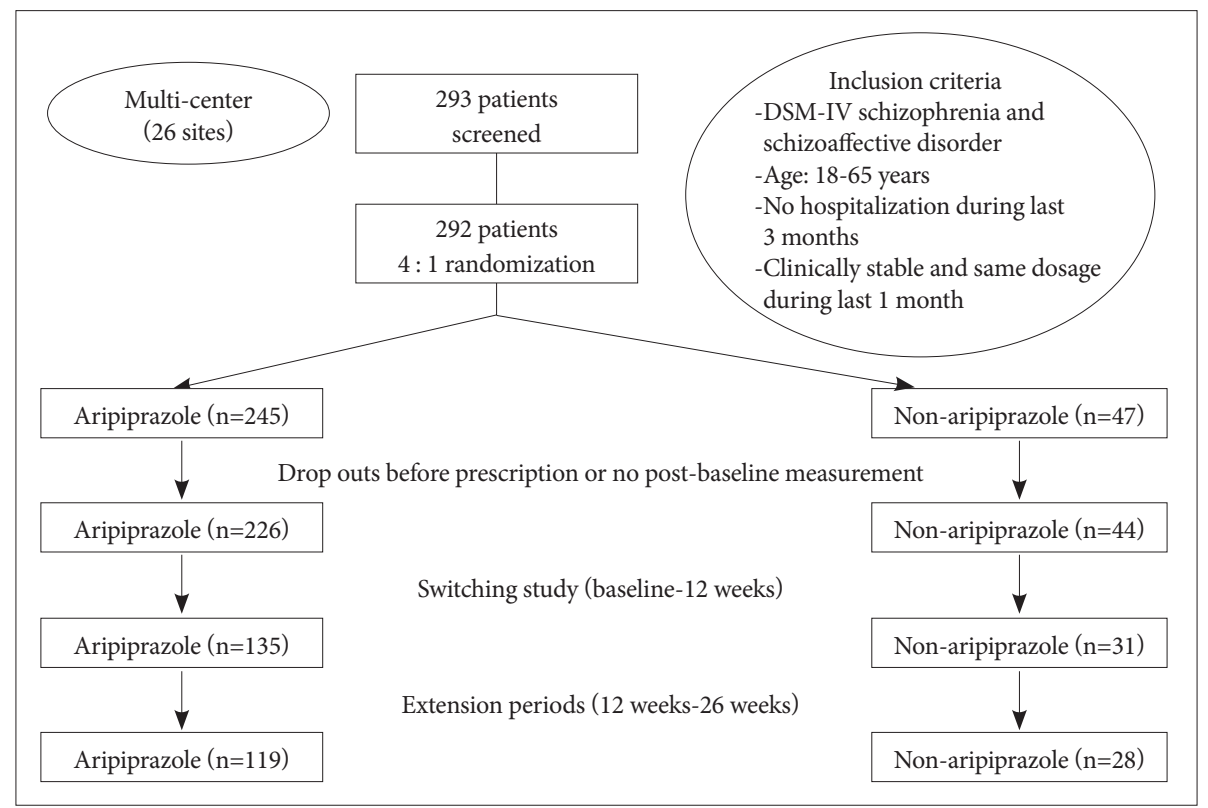

Figure 1. Design overview and enrollment of this study. This extension study was conducted with 166 patients who had completed the switching study and given written consent to the extension study. 
tension study), a written informed consent was again obtained from the patients who agreed to participate in the extension study. Of the 186 patients completing the 12 -week treatment phase, 166 agreed to enroll to long-term extension study. This 14week extension study was approved by respective institutional review boards.

\section{Efficacy and safety measurements}

The symptoms, functions, and adverse events of the subjects were assessed monthly based on the Clinical Global Impression-Improvement/Severity scale (CGI-I/CGI-S), Positive and Negative Syndrome Scale (PANSS), Global Assessment of Functioning (GAF), Udvalg for Kliniske Undersogelser (UKU) side effect rating scale, and treatment-emergent adverse events (TEAEs).

The primary outcome measure in this study was the mean CGI-I score at 26 weeks in the aripiprazole group. The effectiveness analysis consisted of a comparison of the upper bound of the 95\% confidence interval (CI) of the mean CGI-I score to 4 (no change) at the end of the study. The secondary outcome measures included the mean changes from the baseline to the end of the study in the PANSS total score and the CGI-S score. The symptom remission and worsening rates were also estimated at 26 weeks, using the definition of remission and worsening indicated below. Remission was defined as 1) PANSS total score $\leq 60 ; 2$ ) CGI severity score $\leq 3$ (mild); and 3) no rating $\geq 4$ (moderate) on any of the four PANSS psychotic items (delusion, conceptual disorganization, hallucinatory behavior, suspiciousness/persecution). Symptom worsening was defined as 1) $\geq 20 \%$ increase from the baseline in the PANSS total score; 2 ) any change $\geq 2$ points on any of the four PANSS psychotic items (excluding the changes in which the ratings remained at nonpsychotic levels; mild, i.e. $\leq 3$ ); 3) CGI-I score $\geq 6$ (much worse); or 4) discontinuation due to lack of efficacy.

\section{Data analysis}

The baseline clinical and epidemiological characteristics of the aripiprazole and non-aripiprazole groups in this extension study were compared using the Student's t-test for the continuous variables and the $\chi^{2}$ test for the categorical variables. The last-observation-carried-forward (LOCF) convention was used to analyze the mean CGI-I score and the mean changes in the CGI-S and PANSS scores during this extension study.

An upper bound of 95\% CI of the CGI-I score $<4$ at 26 weeks was considered an indicator of effectiveness in the aripiprazole group. The between-group differences in the mean changes in the CGI-S and PANSS scores were evaluated via repeated-measures analysis of covariance (ANCOVA), with the baseline score of each scale as the covariate. The within-group changes from 12 weeks (baseline of the extension study) to 26 weeks were analyzed via the paired t-test or Wilcoxon's signed rank test for the continuous variables and McNemar's test for the categorical variables.

The rate of remission was calculated based on the proportion of patients who met the criteria for remission at 26 weeks relative to 12 weeks. The rate of symptom worsening was calculated, according to the lifetime method, as $100 \times$ number of patients who met the worsening criteria at each visit/[number of observed subjects at each visit-1/2 (number of dropouts between the last visit and the current visit-number of dropouts due to lack of efficacy)]. Tolerability and safety analysis was performed for the patients who were prescribed at least one dose of aripiprazole or another antipsychotic. The occurrence of an adverse event was defined as score $\geq 2$ (moderate) in each item of UKU. Kaplan-Meier survival analysis was performed to estimate the cumulative rate of remission and to investigate the time-to-failure-to-maintain-remission in the patients who met the remission criteria at 12 weeks, the timeto-discontinuation for any cause in all the intention-to-treat (ITT) patients, and the time-to-symptom-worsening in all the ITT patients.

\section{RESULTS}

At the baseline of this extension study, 135 patients were prescribed aripiprazole, and 31 patients were prescribed non-aripiprazole antipsychotics (Fig. 1). The two groups were comparable with respect to their mean ages, gender distribution, baseline PANSS scores, and CGI-S scores (Table 1).

Of 166 patients, 147 (aripiprazole, $n=119$; non-aripiprazole, $\mathrm{n}=28$ ) completed this extension study. The most common reasons for drop outs in the aripiprazole group were lost to followup $(n=6,37.5 \%)$ and lack of efficacy $(n=5,31.3 \%)$.

\section{Efficacy}

At 26 weeks, the LOCF analysis showed that the mean CGII score was $2.92 \pm 1.16$ (95\% CI: $2.72,3.12)$ in the aripiprazole group and $2.81 \pm 1.25$ (95\% CI: $2.35,3.26)$ in the non-aripiprazole group. There was no significant difference between the aripiprazole and non-aripiprazole groups in terms of the changes in their CGI-S scores over time (group $\times$ time interaction, repeated-measures ANCOVA with the score at 12 weeks as the covariate, $\mathrm{p}=0.10$ ). The CGI-S scores were significantly decreased over time, however, in the patients in the non-aripiprazole group, and were not in the aripiprazole group (Fig. 2).

Repeated-measures ANOVA was performed to compare the changes in the PANSS scores over time between the two groups. No significant difference was found in the mean PANSS total score, positive, negative, and general subscale scores in the aripiprazole group, but the mean PANSS total score and positive, 
Table 1. Demographic and clinical characteristics

\begin{tabular}{lccc}
\hline & Aripiprazole & Non-aripiprazole & Total \\
\hline Age (yrs) & $35.7 \pm 9.6$ & $37.2 \pm 11.4$ & $36.0 \pm 9.9$ \\
Gender & & & $0.46^{*}$ \\
Male & $47(34.8 \%)$ & $10(32.3 \%)$ & $57(34.3 \%)$ \\
Female & $88(65.2 \%)$ & $21(67.7 \%)$ & $109(65.7 \%)$ \\
Duration of illness (yrs) & $6.3 \pm 6.3$ & $8.0 \pm 6.2$ & $6.6 \pm 6.3$ \\
Diagnosis & & & $145(87.3 \%)$ \\
Schizophrenia & $116(85.9 \%)$ & $29(93.5 \%)$ & $89(53.6 \%)$ \\
$\quad$ Paranoid type & $72(53.3 \%)$ & $17(54.8 \%)$ & $16(9.6 \%)$ \\
$\quad$ Residual type & $11(8.1 \%)$ & $5(16.1 \%)$ & $3(1.8 \%)$ \\
$\quad 2(1.5 \%)$ & $1(3.2 \%)$ & $0(0 \%)$ \\
Disorganized type & $0(0 \%)$ & $0(0 \%)$ & $37(22.3 \%)$ \\
Catatonic type & $31(23.0 \%)$ & $6(19.4 \%)$ & $21(12.7 \%)$ \\
Sndifferentiated type & $19(14.1 \%)$ & $2(6.5 \%)$ & $0.49^{* *}$ \\
Baseline (12 weeks) PANSS score & & & $49.9 \pm 15.1$ \\
Total score & $49.5 \pm 14.7$ & $51.7 \pm 17.0$ & $11.2 \pm 3.8$ \\
Positive subscale & $11.2 \pm 3.7$ & $10.9 \pm 4.3$ & $12.6 \pm 5.3$ \\
Negative subscale & $12.2 \pm 4.9$ & $26.6 \pm 7.7$ & $26.1 \pm 7.5$ \\
General subscale & $26.0 \pm 7.5$ & $2.7 \pm 1.0$ & $2.6 \pm 0.9$ \\
Baseline (12 weeks) CGI-S & $2.5 \pm 0.8$ & $0.9 \pm 6.5$ & $0.62^{*}$ \\
\hline
\end{tabular}

*analyzed by student t-test, **analyzed by Chi-square test. PANSS: Positive and Negative Syndrome Scale, CGI-S: Clinical Global ImpressionSeverity

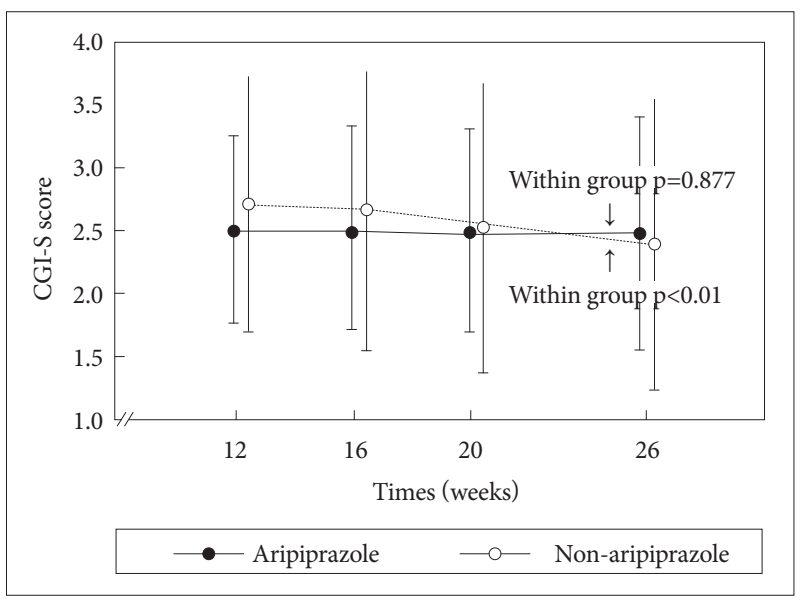

Figure 2. Changes from baseline to endpoint (LOCF). Repeated measures ANOVA was performed for within group comparison (non-aripiprazole group, $n=31, p=0.0014$; aripiprazole group, $n=135, p=0.8689$ ). Repeated measures ANCOVA was performed for between non-aripiprazole group $(n=31)$ and aripiprazole group $(n=135)$ comparison with score of 12 weeks as covariate (group effect, $p=0.0434$; group $\times$ time interaction effect, $p=0.1034$ ).

negative, and general subscale scores significantly decreased over time in the non-aripiprazole group ( $\mathrm{p}<0.01$, all). There was no significant group $\times$ time interaction, however, in the PANSS total and subscale scores ( $\mathrm{p}<0.05$, all).

In the aripiprazole group, the remission rate was $74.8 \%$ $(101 / 135)$ at 12 weeks and $72.6 \%(98 / 135$, dropouts $=16)$ at the end of the study. Of the patients with aripiprazole who were in remission at 12 weeks, $80.2 \%(n=81$, dropouts $=12)$ maintained their remission state until the end of the study. When the patients ( $\mathrm{n}=34)$ with an insufficient response at 12 weeks took aripiprazole continuously without changing their antipsychotics until the endpoint (26 weeks), $32.4 \%$ of these patients $(11 / 34$, dropouts $=4)$ showed further significant symptom improvements and achieved a remission at the endpoint (Fig. 3). The symptom worsening rate at the end of the study was relatively low (6.4\%, life table method).

According to the observed-cases analysis of the aripiprazole group, the overall psychosocial function scores of the patients in the group on the GAF was $69.0 \pm 11.5$ at 26 weeks, and these significantly improved compared to their GAF scores at the baseline of the extension study $(67.1 \pm 11.5, \mathrm{p}<0.05)$.

\section{Safety and tolerability}

Safety analysis was performed on all the patients who were prescribed at least one post-baseline dose of aripiprazole or non-aripiprazole antipsychotics (aripiprazole, $n=131$; non-aripiprazole, $\mathrm{n}=31$ ). Spontaneous TEAEs were reported by 34 (25.2\%) patients on aripiprazole, at least one TEAE from 12 weeks to 26 weeks, and the most commonly reported adverse events included insomnia $(n=6,4.4 \%)$, headache $(n=4,2.9 \%)$, and nausea $(n=4,2.9 \%)$, whereas the most common TEAE 

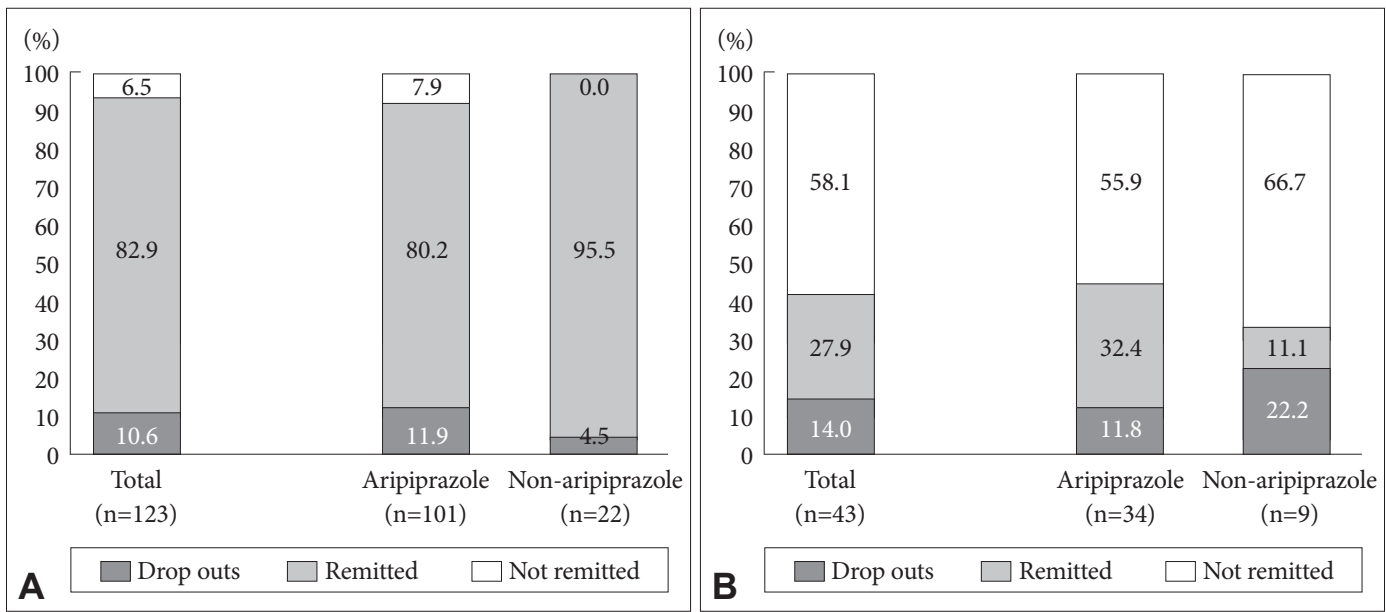

Figure 3. A: Results at endpoint among remitted patients at 12 weeks. About $80-96 \%$ of patients remitted at 12 weeks maintained remission state until endpoint (26 weeks). B: Results at endpoint among the patients who had not remitted at 12 weeks. About $11-32 \%$ of patients who had not remitted at 12 weeks were converted to remission state at endpoint.

Table 2. Comparisons UKU adverse event (neurologic adverse events) within group and between-groups

\begin{tabular}{|c|c|c|c|c|c|c|c|c|}
\hline \multirow{2}{*}{ UKU } & & \multicolumn{3}{|c|}{ Aripiprazole } & \multicolumn{3}{|c|}{ Non-aripiprazole } & \multirow{2}{*}{$\begin{array}{c}\text { Between group } \\
\text { p-value** }\end{array}$} \\
\hline & & $\mathrm{N}$ & Mean \pm SD & p-value* & $\mathrm{N}$ & Mean \pm SD & p-value* & \\
\hline \multirow[t]{2}{*}{ Dystonia } & 12 weeks & 135 & $0.07 \pm 0.40$ & 0.31 & 31 & $0.16 \pm 0.45$ & 0.50 & 0.05 \\
\hline & 26 weeks & 119 & $0.04 \pm 0.30$ & & 28 & $0.11 \pm 0.31$ & & \\
\hline \multirow[t]{2}{*}{ Rigidity } & 12 weeks & 135 & $0.03 \pm 0.17$ & 0.25 & 31 & $0.03 \pm 0.18$ & 0.99 & 0.64 \\
\hline & 26 weeks & 119 & $0.01 \pm 0.09$ & & 28 & $0.00 \pm 0.00$ & & \\
\hline \multirow[t]{2}{*}{ Hypokinesia/Akinesia } & 12 weeks & 135 & $0.13 \pm 0.40$ & $<0.01$ & 31 & $0.16 \pm 0.37$ & 0.13 & 0.90 \\
\hline & 26 weeks & 119 & $0.04 \pm 0.20$ & & 28 & $0.04 \pm 0.19$ & & \\
\hline \multirow[t]{2}{*}{ Hyperkinesia } & 12 weeks & 135 & $0.01 \pm 0.09$ & 0.99 & 31 & $0.00 \pm 0.00$ & 0.99 & 0.26 \\
\hline & 26 weeks & 119 & $0.01 \pm 0.09$ & & 28 & $0.04 \pm 0.19$ & & \\
\hline \multirow[t]{2}{*}{ Tremor } & 12 weeks & 135 & $0.13 \pm 0.38$ & 0.18 & 31 & $0.10 \pm 0.30$ & 0.99 & 0.88 \\
\hline & 26 weeks & 119 & $0.09 \pm 0.32$ & & 28 & $0.11 \pm 0.42$ & & \\
\hline \multirow[t]{2}{*}{ Akathisia } & 12 weeks & 135 & $0.12 \pm 0.41$ & 0.02 & 31 & $0.06 \pm 0.25$ & 0.99 & 0.96 \\
\hline & 26 weeks & 119 & $0.04 \pm 0.24$ & & 28 & $0.04 \pm 0.19$ & & \\
\hline \multirow[t]{2}{*}{ Amenorrhea } & 12 weeks & 84 & $10.7 \%$ & $0.01^{\dagger}$ & 16 & $31.2 \%$ & 0.32 & $0.15^{\ddagger}$ \\
\hline & 26 weeks & 70 & $2.8 \%$ & & 16 & $12.5 \%$ & & \\
\hline
\end{tabular}

*comparisons between 12 weeks and 26 weeks using Wilcoxon signed rank test, ${ }^{*}$ comparisons at end point between groups using Wilcoxon rank sum test , ${ }^{\dagger}$ comparisons between 12 weeks and 26 weeks using McNemar's test, ${ }^{\ddagger}$ comparisons at end point between groups using Fisher’s exact test. UKU: Udvalg for Kliniske Undersogelser side effect rating scale

in the non-aripiprazole group was dystonia $(n=6,2.5 \%)$. In the aripiprazole group, there were two serious adverse events (SAEs): suicide attempt and aggravation of psychotic disorder with no causal relationship between two SAEs and aripiprazole. There was no SAE in the non-aripiprazole group.

When the scores of the patients in the aripiprazole group on each item of UKU at 12 and 26 weeks were compared, the scores on hypokinesia, akathisia, and amenorrhea were found to have significantly decreased over time (Table 2). When adverse events were defined as a score $\geq 2$ on each item of UKU, a decrease in the prolactin-related adverse events and akathisia at the endpoints $(\mathrm{p}<0.05)$ was found in the aripiprazole gr- oup (Table 2).

\section{DISCUSSION}

This study suggests that most clinically stable outpatients with schizophrenia maintain their remission states after being switched to aripiprazole, without serious symptom aggravation and adverse events over the course of 26 weeks of treatment. It was shown in this study that $80.2 \%(81 / 101)$ of the subjects maintained their remission state from 12 to 26 weeks, without symptom aggravation. The symptom worsening rate at the end of the study was $6.4 \%$. Moreover, $32.4 \%$ of the patients with an 
insufficient response at 12 weeks experienced further improvement and achieved remission by 26 weeks when they took aripiprazole continuously without changing their antipsychotic medication until the endpoint.

When we compared the results of our study with those of other naturalistic studies such as BETA, EU-BETA and the clinical antipsychotic trials of intervention effectiveness (CATIE) although there were some differences in the definition of response and duration of the studies, the CGI-I score of our study at 26 weeks (2.92, 95\% CI: 2.72-3.12) was similar to those of BETA (2.77, 95\% CI 2.68-2.86) and EU-BETA (3.16, 95\% CI 3.04-3.28) at 8 weeks ${ }^{10,11}$ and the discontinuation rate of treatment for any cause of our study at 26 weeks was slightly higher than that of CATIE study ( $49.7 \%$ vs. $40 \%){ }^{12}$

As mentioned above, among the patients who achieved a remission state at 12 weeks, approximately $20 \%$ did not maintain the remission. These findings are consistent with the previous findings that approximately $20 \%$ of the patients receiving aripiprazole or SGAs relapsed within six months of their treatment. ${ }^{13,14}$

We were interested in assessing the rate of symptom worsening, defined based on the PANSS total score and the psychotic item scores, CGI-I, and drop out due to lack of efficacy. These criteria (with the exception of CGI-I) were used in our previously published study on a risperidone long-acting injection formulation. ${ }^{15}$ The symptom worsening rate after switching to aripiprazole was relatively low in our previous published study $\left(12.4 \%\right.$ at 12 weeks), ${ }^{9}$ and this rate remained low until 26 weeks (6.4\%) in spite of the use of more stringent criteria for symptom worsening, including "drop out due to an insufficient response." Considering the low rate (6.4\%) of symptom worsening and the relatively high (72.6\%) remission rate at the endpoint (26 weeks), our results suggest that switching to aripiprazole from other antipsychotics is effective and tolerable in clinically stable patients with schizophrenia.

Interestingly, of the patients who did not meet the remission criteria at the baseline of the extension study, 32.4\% developed remission by the 26 th-week follow-up while $7.9 \%$ of the patients in remission at the baseline no longer met the remission state.

According to the results of our study on adverse events, the patients who switched to aripiprazole experienced less adverse events at the endpoint, such as hypokinesia, akathisia, and amenorrhea, compared with 12 weeks. These results are consistent with findings from other studies: that aripiprazole is associated with fewer EPSs or prolactin elevation. ${ }^{1,4}$ Apart from EPS- and prolactin-related adverse events, aripiprazole was found to be associated with fewer metabolic side effects such as weight gain or increased lipid levels..$^{5-7}$ Likewise, our study showed that there was no significant change in the blood glucose or lipid levels in both groups for the duration of the extension study.

This study has a number of limitations common to a naturalistic open, observational study: 1) The absence of a placebo control group made it difficult to confirm whether the rate of symptom worsening in the patients who were prescribed aripiprazole was lower than that in the patients with no medication. The rate of symptom worsening of this extension study was $7.9 \%$ at 14 weeks (about $2.6 \%$ per month). According to the previous reports that the monthly relapse rates were estimated to be $3.5 \%$ per month for the patients on maintenance neuroleptics and $11.0 \%$ per month for the patients who had discontinued their medication, ${ }^{14}$ the lower symptom worsening rate in this study may be due to the maintenance efficacy of aripiprazole rather than to the natural course of the disease. 2) As this study is an acute-phase extension study, 20 of the 186 subjects who completed the acute-phase study did not agree to participate in the extension study. This might have affected the study results.

In conclusion, this study showed that the effectiveness of aripiprazole after a switch from other antipsychotics was maintained until 26 weeks. Our results also confirmed that the clinically stable patients with schizophrenia were successfully switched from their existing antipsychotics to aripiprazole until 26 weeks, without symptom worsening or serious adverse events.

\section{Acknowledgments}

This study was supported by Korea Otsuka Pharmaceuticals (KOP 010402).

\section{REFERENCES}

1. Kane JM, Carson WH, Saha AR, McQuade RD, Ingenito GG, Zimbroff DL, et al. Efficacy and safety of aripiprazole and haloperidol versus placebo in patients with schizophrenia and schizoaffective disorder. J Clin Psychiatry 2002;63:763-771.

2. Potkin SG, Saha AR, Kujawa MJ, Carson WH, Ali M, Stock E, et al. Aripiprazole, an antipsychotic with a novel mechanism of action, and risperidone vs placebo in patients with schizophrenia and schizoaffective disorder. Arch Gen Psychiatry 2003;60:681-690.

3. Pigott TA, Carson WH, Saha AR, Torbeyns AF, Stock EG, Ingenito GG. Aripiprazole for the prevention of relapse in stabilized patients with chronic schizophrenia: a placebo-controlled 26-week study. J Clin Psychiatry 2003;64:1048-1056.

4. Kasper S, Lerman MN, McQuade RD, Saha A, Carson WH, Ali M, et al. Efficacy and safety of aripiprazole vs. haloperidol for long-term maintenance treatment following acute relapse of schizophrenia. Int J Neuropsychopharmacol 2003;6:325-337.

5. Chrzanowski WK, Marcus RN, Torbeyns A, Nyilas M, McQuade RD. Effectiveness of long-term aripiprazole therapy in patients with acutely relapsing or chronic, stable schizophrenia: a 52-week, open-label comparison with olanzapine. Psychopharmacology (Berl) 2006;189:259-266.

6. Marder SR, McQuade RD, Stock E, Kaplita S, Marcus R, Safferman AZ, et al. Aripiprazole in the treatment of schizophrenia: safety and tolerability in short-term, placebo-controlled trials. Schizophr Res 2003;61: 123-136. 
7. Kolotkin RL, Corey-Lisle PK, Crosby RD, Kan HJ, McQuade RD. Changes in weight and weight-related quality of life in a multicentre, randomized trial of aripiprazole versus standard of care. Eur Psychiatry 2008; 23:561-566.

8. Findling RL, Robb A, Nyilas M, Forbes RA, Jin N, Ivanova S, et al. A multiple-center, randomized, double-blind, placebo-controlled study of oral aripiprazole for treatment of adolescents with schizophrenia. Am J Psychiatry 2008;165:1432-1441.

9. Kim CY, Chung S, Lee JN, Kwon JS, Kim do H, Kim CE, et al. A 12week, naturalistic switch study of the efficacy and tolerability of aripiprazole in stable outpatients with schizophrenia or schizoaffective disorder. Int Clin Psychopharmacol 2009;24:181-188.

10. Tandon R, Marcus RN, Stock EG, Riera LC, Kostic D, Pans M, et al. A prospective, multicenter, randomized, parallel-group, open-label study of aripiprazole in the management of patients with schizophrenia or schizoaffective disorder in general psychiatric practice: Broad Effectiveness Trial With Aripiprazole (BETA). Schizophr Res 2006;84:77-89.

11. Wolf J, Janssen F, Lublin H, Salokangas RK, Allain H, Smeraldi E, et al. A prospective, multicentre, open-label study of aripiprazole in the man- agement of patients with schizophrenia in psychiatric practice in Europe: Broad Effectiveness Trial with Aripiprazole in Europe (EU-BETA). Curr Med Res Opin 2007;23:2313-2323.

12. Lieberman JA, Stroup TS, McEvoy JP, Swartz MS, Rosenheck RA, Perkins DO, et al. Effectiveness of antipsychotic drugs in patients with chronic schizophrenia. N Engl J Med 2005;353:1209-1223.

13. Moeller KE, Shireman TI, Liskow BI. Relapse rates in patients with schizophrenia receiving aripiprazole in comparison with other atypical antipsychotics. J Clin Psychiatry 2006;67:1942-1947.

14. Weiden PJ, Olfson M. Cost of relapse in schizophrenia. Schizophr Bull 1995;21:419-429.

15. Kim CY, Shin YW, Joo YH, Hong JP, Lee GH, Choi SK. Risperidone dosing pattern and clinical outcome in psychosis: an analysis of 1713 cases. J Clin Psychiatry 2005;66:887-893.

16. Tandon R, Belmaker RH, Gattaz WF, Lopez-Ibor JJ Jr, Okasha A, Singh B, et al. World Psychiatric Association Pharmacopsychiatry Section statement on comparative effectiveness of antipsychotics in the treatment of schizophrenia. Schizophr Res 2008;100:20-38. 